\title{
Cinéma et modernité chez Siegfried Kracauer
}

\author{
Par OLIVER AGARD \\ Université de Paris 4 Sorbonne
}

De même que Kracauer déniait volontiers sa formation d'architecte, il ne souhaitait pas être considéré comme un spécialiste de cinéma, et expliquait souvent qu'à travers le cinéma, c'était autre chose qui était visé dans ses écrits. Dans sa correspondance avec Adorno, il insiste par exemple sur l'importance du chapitre final de la Théorie du film, qui est pour lui l'aboutissement et l'enjeu de ce livre ${ }^{1}$. De fait, le cinéma est sur un plan méthodologique et thématique au cœur de la démarche d'ensemble de Kracauer, et c'est ce qui explique aussi le lien étroit qu'il entretient avec la question de la ville, autre thème central pour cet auteur. Cinéma et ville sont cependant deux voies d'accès à une question plus fondamentale encore, qui court dans toute l'œuvre de Kracauer, à savoir la question de la modernité, l'originalité de Kracauer étant qu'il pense cette question à travers les objets du monde, les phénomènes, et non pas à travers de grandes théories sociologiques sur le processus de modernisation. À travers ces deux thèmes (ville et cinéma) et leur relation, il est donc possible de donner une idée de l'itinéraire intellectuel de Kracauer, et c'est ce que j'aimerais faire ici, en insistant aussi sur un troisième thème que l'on voit prendre une importance croissante dans les écrits de Kracauer sur le cinéma, celui de la vie, qui devient clairement dominant dans la grande Théorie du film de 1960.

${ }^{1}$ Voir par exemple la lettre du 16.01.1964 à Adorno: Theodor W. Adorno \& Siegfried Kracauer, Briefwechsel 1923-1966, Frankfurt/Main, Suhrkamp, 2008, p. 639-640. 


\section{La ville mélancolique}

La réflexion de Kracauer sur la ville semble au départ marquée par les textes de Georg Simmel, en particulier la deuxième partie de la Philosophie de l'argent ${ }^{1}$, l'essai sur « Les grandes villes et la vie de l'esprit ${ }^{2}$, et la digression, ou l'excursus sur l'étranger ${ }^{3}$ (extrait de la grande sociologie de 1907). Selon Simmel, la mentalité urbaine est le produit de l'abstraction moderne, qui se traduit chez le citadin par l'intellectualisme (qui est le corollaire de l'intensification de la vie nerveuse, base psychologique de la vie citadine), par une manière prosaïque de traiter les hommes et les choses (d'où résulte l'impersonnalité des échanges), par un rapport quantitatif au temps, une attitude blasée (qui est aussi une indifférence envers la qualité des choses), et une posture mentale faite de réserve et d'antipathie latente. La métropole, tout en étant le produit d'une dynamique de dépersonnalisation et d'abstraction, apparaît néanmoins chez Simmel aussi comme un laboratoire de formes nouvelles d'identité: le nivellement quantitatif suscite dialectiquement l'aspiration à se singulariser, au sens de l'individualité qualitative. La métropole n'en reste pas moins le lieu d'un conflit, conflit entre «culture subjective» et "culture objective», qui apparaît finalement à Simmel comme un conflit inhérent à la vie même, dans la mesure où celle-ci ne peut s'exprimer que dans des formes qui finissent par se retourner contre elle, dans une sorte de tragédie de la culture ${ }^{4}$.

Dans ses textes de jeunesse (aujourd'hui rassemblés dans les deux volumes des Frühe Schriften ${ }^{5}$ ), imprégnés de Kulturkritik, critique de la civilisation moderne, Kracauer reprend ce thème de la tragédie de la culture, et semble ne retenir que le versant négatif de la vision simmélienne, l'idée de la ville comme aliénation existentielle, rupture avec la communauté (Gemeinschaft), site d'un désenchantement du monde, absence d'un abri transcendantal. Par la suite, les textes de la première moitié des années 1920

${ }^{1}$ Georg Simmel, Philosophie de l'argent, trad. fr. S. Cornille et P. Ivernel Paris, PUF, 1987 (voir en particulier le chapitre 6, « le style de vie », p. 545-662).

${ }^{2}$ Georg Simmel, «Les Grandes villes et la vie de l'esprit», dans Id., Philosophie de la modernité, trad. fr. J.-L. Vieillard-Baron, Paris, Éditions Payot \& Rivages, 1989, p. 233-252.

Georg Simmel, «Excursus sur l'étranger», dans Id., Sociologie. Études sur les formes de la socialisation, Paris, PUF, 1999, p. 663-668.

${ }^{4}$ Cf. Georg Simmel, « Le Conflit de la culture moderne », dans Id., Philosophie de la modernité, op. cit., p. 229-260.

5 Siegfried Kracauer, Frühe Schriften aus dem Nachlass (Werke 9.1 et 9.2), Frankfurt/Main, Suhrkamp, 2004. 
restent marqués par cette thématique existentialiste de l'isolement du citadin dans un monde désenchanté. Pour Kracauer, « ceux qui attendent» («Die Wartenden »), et qui souffrent en silence du vide spirituel, sont justement des citadins, qui «passent le plus souvent leurs journées dans la solitude des grandes villes $»^{1}$. Les images de l'exil et de l'errance de l'étranger restent chez Kracauer opératoires, même quand sa pensée se fait plus " mondaine », dans ce texte beaucoup plus politique qu'est Les Employés.

La ville est marquée par la mélancolie, en tant que lieu de passage où rien ne dure. L'espace urbain, où évoluent les citadins nomades, est donc présenté comme un espace de transit, à l'image de la gare, définie comme « un lieu qui n'est pas un site » («ein Ort, der keine Stätte ist $»^{2}$ ). Le paradigme de ces espaces de transit, de ces «non-lieux » ${ }^{3}$ est la rue : à l'image de la ville, elle est un espace contingent, ouvert à une infinité de parcours aléatoires. Comme le note Pierre Sansot: «La ville nous fait libre, libre d'aller où bon nous semble et à l'heure qui nous convient, dans un espace où tous les chemins sont possibles $\gg{ }^{4}$. Un autre lieu de transit et non-lieu anonyme, est la gare ou l'hôtel. En tant que lieu de passage, la ville n'a pas de mémoire, à l'image du Kurfürstendamm, « rue sans mémoire » : «Si bien des rues semblent avoir été tracées pour l'éternité, l'actuel Kurfürstendamm est l'incarnation du flot vide du temps, à l'intérieur duquel rien n'est susceptible de durer $»^{5}$.

La ville, théâtre d'interactions fugaces, de constellations toujours renouvelées est en mouvement incessant. Le paysage urbain est donc éphémère, et lui-même en transit : les chantiers voisinent avec les ruines. Par ailleurs, le vide est omniprésent, les rues sont des trouées sans fin. Berlin est caractérisée par ses grands espaces, ses perspectives à perte de vue, et l'infinité de cette étendue est anxiogène. La mélancolie du paysage se communique au citadin, qui est de toute façon lui-même prédisposé à la

${ }^{1}$ Siegfried Kracauer, «Ceux qui attendent», dans Id., L'Ornement de la masse, Olivier Agard et Philippe Despoix (éd.), trad. fr. S. Cornille, Paris, Éditions La Découverte, 2007, p. 107.

${ }^{2}$ Siegfried Kracauer, «Die Eisenbahn », dans Id., Essays, Feuilletons, Rezensionen 1928-1931 (Werke 5.3, herausgegeben von Inka Mülder-Bach ; unter Mitarbeit von Sabine Biebl), Berlin, Suhrkamp, 2011, p. 208.

${ }^{3}$ Nous empruntons cette catégorie à Marc Augé, Non-lieux: Introduction à une anthropologie de la surmodernité, Paris, Éditions du Seuil, 1992.

${ }^{4}$ Pierre Sansot, Poétique de la ville. Paris, Éditions Klincksieck, 1971, p. 392.

5 Siegfried Kracauer, Rues de Berlin et d'ailleurs, trad. fr. J.-F. Boutout, Paris, Éditions Belles lettres, 2013, p. 27. 
mélancolie. Le texte de Kracauer intitulé « Ennui » ${ }^{1}$, qui inaugurait en 1924 les «textes de ville» et analysait ce que Pierre Sansot appelle ailleurs $l^{\prime}$ « angoisse des dimanches en ville $»^{2}$, peut se lire comme une variation de ce thème simmélien de la mélancolie du blasé, mais là aussi avec un infléchissement existentialiste. Dans ce texte aux accents pascaliens, l'ennui, pratiqué de façon presque méthodique, est ce qui permet d'accéder à un sentiment de l'existence, à une prise de conscience de la vacuité de l'activité quotidienne dans un monde rationalisé, vacuité que les divertissements modernes essaient de remplir en nous plongeant dans le rêve et le simulacre.

Il importe toutefois déjà de comprendre que cette mélancolie que Kracauer met en évidence par le biais d'une phénoménologie de la métropole, et qui est donc aussi celle de son regard, n'est pas une nostalgie de l'enracinement. Il n'y a aucune perspective d'un retour en arrière, et on ne trouvera pas chez Kracauer d'apologie de la ruralité ou du lien organique avec le peuple et le terroir. Kracauer ne tient pas le discours de la Heimat, du sol et du sang. La mélancolie est le principe d'un rapport critique à la réalité désenchantée du monde, qu'on ne peut pas ne pas affronter, en dépit de sa dimension négative. Même si Kracauer développe par la suite un rapport plus positif à la ville, à la mobilité citadine, au moment où sa pensée devient moins existentialiste et plus politique, au milieu des années 1920, cette mélancolie demeure, en tant qu'instrument cognitif et refus d'adhérer à un modernisme sans profondeur. C'est ce qui distingue la conception que se fait Kracauer du "nomadisme» de sa version actuelle, le nomadisme étant aujourd'hui presque devenu un mot d'ordre du système (le travailleur moderne est sommé d'être mobile). Dans le monde tel qu'il est, on ne peut accéder, pour Kracauer, à une forme de réalité qu'en passant par l'irréalité, et il est plus pertinent de flâner dans les halls d'hôtels, de lire des romans policiers et d'aller au cinéma que de vouloir redonner vie aux textes sacrés, comme le font Buber et Rosenzweig, dont Kracauer critique sévèrement la traduction de la Bible, dans un texte qui marque le passage à une phase plus politique de sa pensée ${ }^{3}$.

On peut être tenté de parler au sujet de la démarche de Kracauer d'une sorte de phénoménologie de la surface, mais il faut bien noter que Kracauer récuse d'emblée la possibilité d'accéder à des essences. Dans l'essai de 1922 La Sociologie comme science (Soziologie als Wissenschaft), il arrive à la

\footnotetext{
${ }^{1}$ Siegfried Kracauer, « Ennui », dans Id., L'Ornement de la masse, op. cit., p. 60-71.

${ }^{2}$ Pierre Sansot, Poétique de la ville, op. cit., p. 392.

${ }^{3}$ Siegfried Kracauer, «La Bible en allemand », dans Id., L'Ornement de la masse, op. cit., p. 163-175.
} 
conclusion qu'il est impossible d'étendre la phénoménologie au monde social, celui-ci étant sous-déterminé, délaissé par l'esprit. Dans son ouvrage sur Le Roman policier (Der Detektivroman) de 1925/26 ${ }^{1}$, il estime qu'on ne peut plus atteindre l'inconditionné si ce n'est sur un mode négatif comme reflet d'une réalité perdue.

\section{Le cinéma, médium de la ville}

Le cinéma apparaît pour Kracauer comme le médium adéquat de cet espace « désacralisé $~^{2}$ qu'est la ville, et c'est le point que développe longuement la critique du film Die Straße de Karl Grune. Ce texte montre bien comment se noue pour Kracauer l'articulation entre la ville et le cinéma. Le cinéma semble avoir pour Kracauer l'éminent mérite de montrer le monde moderne dans sa réalité, il est une mimèsis de la négativité. En cela, il est structurellement comparable au roman policier. Le film Die Straße montre ainsi un monde qui n'offre plus d'abri transcendantal à l'individu : la ville et la rue sont sans «connexion" (Verknüpfung) avec le divin: "Le film La rue actuellement projeté dans de nombreuses villes en Allemagne dépeint un monde dans lequel l'œuvre de la liaison ne s'accomplit pas $\rangle^{3}$. Kracauer ajoute que les individus y apparaissent comme des atomes qui coexistent sans plus être ensemble, qui s'accouplent plus qu'ils ne s'aiment. Ce film, sur lequel Kracauer reviendra à plusieurs reprises (en particulier dans la première version de la Théorie du film, rédigée à Marseille en 1940/41), raconte comment un petit-bourgeois quitte son foyer confortable et étouffant pour la rue, en quête d'une expérience qui lui fasse surmonter sa mélancolie (due, selon Kracauer, à la perte des idéaux, et des valeurs ${ }^{4}$ ). Il se retrouve accusé du meurtre d'un client d'une prostituée : il est innocenté in extremis, et retourne piteusement dans son foyer. La vision de la ville développée dans ce film, comme univers chaotique, anarchique, livré au crime, et aux pulsions,

${ }^{1}$ Siegfried Kracauer, Le Roman policier, trad. fr. G. et R. Rochlitz, Paris, Éditions Payot \& Rivages, 1981.

${ }^{2}$ Pierre Sansot, Poétique de la ville, op. cit., p. 42-46.

${ }^{3}$ Siegfried Kracauer, « Der Künstler in dieser Zeit», dans Id., Essays, Feuilletons, Rezensionen 1924-1927 (Werke 5.2, herausgegeben von Inka Mülder-Bach; unter Mitarbeit von Sabine Biebl), Berlin, Suhrkamp, 2011, p. 236 [Der Morgen, Avril 1925].

${ }^{4} C f$. le résumé du film dans Siegfried Kracauer, "Marseiller Entwurf», dans $I d$., Theorie des Films. Die Errettung der äußeren Wirklichkeit (Werke 3), Frankfurt/ Main, Suhrkamp, 2005, p. 699. 
n'est pas sans rappeler les lithographies du cycle «Stadtnacht» de Max Beckmann, dont Kracauer a par ailleurs livré un commentaire ${ }^{1}$. À l'image des textes de ville de Kracauer, le film ne propage pas la nostalgie d'un ordre révolu : la famille petite-bourgeoise n'est pas présentée dans le film comme un univers positif, mais plutôt comme un environnement étriqué, stérile, mortifère : le retour au bercail est teinté d'amertume.

Dans la Rue, le cinéma semble donc avoir en premier lieu une fonction de révélateur du négatif, et beaucoup d'autres textes de Kracauer vont à l'époque dans le sens de cette thèse : ainsi, les films de Chaplin, comme Le Cirque, donneraient à voir le morcellement du monde mécanisé et sans âme, dans lequel l'individu est en proie à l'agression permanente de l'organisation $^{2}$. Kracauer trouve par la suite cette thématique jusque chez Harold Lloyd, René Clair ou dans le film Le Joueur d'échec de Raymond Bernard ${ }^{3}$.

S'il y a une affinité entre cinéma et ville, cela tient à la spécificité du langage cinématographique. Tout d'abord, le cinéma est un art visuel, or la ville est dominée par les signaux optiques : lumières, feux de circulation, enseignes et publicités lumineuses. Simmel caractérisait déjà le citadin par l'hypertrophie du regard. Le regard était selon lui le plus abstrait de tous les sens, et l'abstraction croissante de l'économie monétaire, dans la mesure où elle instituait un rapport médiat au monde, tendait donc à favoriser le développement du sens optique.

En tant que captation visuelle de l'apparence des choses, le cinéma est le mieux placé pour rendre compte d'un environnement urbain tout entier tourné vers l'extériorité, décentré, dépourvu d'unité organique, de cohérence. Dans ses critiques de film des années 1920, dans lesquelles s'ébauche déjà une réflexion sur la théorie du film, Kracauer relève ainsi à plusieurs reprises

\footnotetext{
${ }^{1}$ Siegfried Kracauer, « Max Beckmann », dans Id., Essays, Feuilletons, Rezensionen 1906-1923 (Werke 5.1, herausgegeben von Inka Mülder-Bach ; unter Mitarbeit von Sabine Biebl), Berlin, Suhrkamp, 2011, p. 233-239 [Die Rheinlande, Vol. 21, juillet 1921].

${ }^{2}$ Siegfried Kracauer, « Chaplin : zu seinem Film Zirkus », dans Id., Kleine Schriften zum Film 1928-1931 (Werke 6.2, hrsg. von Inka Mülder-Bach; unter Mitarb. von Mirjam Wenzel und Sabine Biebl), Frankfurt/Main, Suhrkamp, 2004, p. 33 [FZ, 15.2.1928]. Voir également Siegfried Kracauer, "Zwei Jongleure », dans Id., Kleine Schriften zum Film 1928-1931, op. cit., p. 513-515 [FZ, 18.6.1931].

3 Siegfried Kracauer, "Der Schachspieler», dans Id., Kleine Schriften zum Film 1921-1927 (Werke 6.1, hrsg. von Inka Mülder-Bach ; unter Mitarb. von Mirjam Wenzel und Sabine Biebl), Frankfurt/Main, Suhrkamp, 2004, p. 285-286 [FZ, 24.1.1927].
} 
que le cinéma est l'art de l'aléatoire ${ }^{1}$ et de l'association libre, et qu'il rend ainsi compte d'une réalité fragmentée, kaléidoscopique où tout se joue à la surface, sans régulation transcendante. C'est pourquoi le trafic est une figure à la fois éminemment urbaine et éminemment cinématographique. Un sujet filmique est ainsi l'agent de la circulation, un acteur central du trafic, dont le rôle est décrit dans le texte intitulé "Les Chauffeurs saluent ${ }^{2}$. Les contrastes et le chaos qu'offre la surface de la ville sont tout à fait conformes à l'esprit du film, tout comme la mélancolie de ces paysages promis à la destruction. La caméra peut rendre compte de cette surface contingente et éclatée, non seulement parce qu'elle est une captation de la surface, mais aussi parce qu'elle a la capacité de se mouvoir librement dans un univers polycentré, qui ne peut plus être embrassé par un point de vue surplombant et global $^{3}$. Elle nous permet ainsi de pénétrer au cœur des masses de la métropole. La déambulation dans la ville est donc pour Kracauer un thème éminemment filmique ${ }^{4}$, et la caméra de René Clair est tout à fait comparable à un flâneur dans les rues de la ville .

Cette idée du cinéma comme exposition négative d'un monde dissocié explique le désaccord - très net au départ - de Kracauer avec Balász, dont la théorie, au moins dans L'Homme visible (Der sichtbare Mensch) est marquée par la philosophie de la vie d'un Bergson, envers laquelle Kracauer ne partageait pas l'enthousiasme de Simmel. En effet, pour Balász, le cinéma exprime « l'aspiration de l'être humain dans une civilisation intellectualisée à l'excès et devenue abstraite, à l'expérience vécue d'une réalité concrète, non médiatisée, qui ne passe pas par le filtre des idées et des mots ${ }^{6}$. Kracauer estime en revanche que ce que montre le film est le contraire d'un tournant vers la vraie concrétude : ce qu'il capte est le produit de la mauvaise rationa-

${ }^{1}$ Par exemple Siegfried Kracauer, «Ein Seefilm», dans Id., Kleine Schriften zum Film 1921-1927, op. cit., p. 187 [FZ, 14.1.1926].

2 Siegfried Kracauer, "Les Chauffeurs saluent», dans Id., Le Voyage et la danse : Figures de ville et vues de films, trad. fr. S. Cornille, Saint-Denis, PUV, 1996, p. 32$33[F Z, 20.8 .1926]$.

${ }^{3}$ Siegfried Kracauer, «Film-Notizen », dans Id., Kleine Schriften zum Film 19321961 (Werke 6.3, hrsg. von Inka Mülder-Bach ; unter Mitarb. von Mirjam Wenzel und Sabine Biebl), Frankfurt/Main, Suhrkamp, 2004, p. 65 [FZ, 3.6.1932].

${ }^{4}$ Siegfried Kracauer, «Von Kindern, Affen und jungen Hunden », dans Id., Kleine Schriften zum Film 1921-1927, op. cit., p. 96-97 [FZ, 18.10.1924].

${ }^{5}$ Siegfried Kracauer, «Idyll, Volkserhebung, Charakter », dans Id., Kleine Schriften zum Film 1932-1961, op. cit., p. 130-137 [FZ, 24.1.1933].

${ }^{6}$ Bela Balázs, L'Homme visible et l'esprit du cinéma, Belval, Éditions Circé, 2010, p. 135-136. 
lité de la pensée capitaliste, et il présente justement l'intérêt de nous la montrer sans détour ${ }^{1}$. Le cinéma se déploie dans un temps-espace qui est celui de la ville, et dont Lukács explique à peu près à la même époque que c'est le temps parcellaire du capitalisme mécanisé ${ }^{2}$. Si Kracauer recourt aux catégories de Bergson (le temps-espace opposé au temps-durée), c'est donc dans un sens inverse de celui de Balázs.

Le grand texte de 1927 sur la photographie est consacré à cette capacité de révélation des médias photographiques en tant que nouveau mode de représentation, mais il introduit une nuance frappante entre photographie et cinéma. Kracauer part dans cet essai de la photographie d'une jeune « diva du cinéma» de vingt-quatre ans qu'il compare à celle d'une femme de quatre-vingt ans, aujourd'hui grand-mère, prise plus de soixante ans auparavant dans un atelier. Vue aujourd'hui, cette grand-mère a l'apparence d'une poupée, à la fois ridicule et un peu effrayante, d'une simple collection de détails décoratifs démodés : «Sous les yeux des petits-enfants, la grand-mère se dissout en détails à la mode-démodés. Les petits-enfants rient de ce costume resté maître du terrain après l'évanescence de la personne qui le portait - une décoration extérieure qui s'est autonomisée - ; ils sont sans piété, et aujourd'hui les jeunes filles s'habillent autrement. Ils rient, tout en étant parcourus d'effroi $»^{3}$. Ces détails sont juxtaposés les uns aux autres : là où le souvenir que nous avons de la grand-mère cristallise tout un vécu et confère un sens à son image, son portrait photographique est une pure collection de détails co-existants dans l'espace, la photographie capte de façon en partie impersonnelle une série de traces, sans leur donner une signification. Derrière cette opposition, il y a à nouveau la distinction bergsonienne entre temps-durée et temps-espace.

Comme le cinéma dans les textes évoqués plus haut, la photographie semble avoir donc un rôle de révélation de la négativité du monde pris dans le temps-espace : elle donne à voir en premier lieu la contingence de l'ordre fantomatique qui se fait passer pour la réalité : avec le recul historique, nous nous apercevons que l'univers capté par l'objectif, c'est-à-dire le nôtre, n'est qu'une constellation arbitraire : «Cette réalité fantomatique est non délivrée. Elle est constituée de parties de l'espace dont la composition est si peu

${ }^{1}$ Voir Siegfried Kracauer, « Bücher vom Film », dans Id., Kleine Schriften zum Film 1921-1927, op. cit., p. 371-372.

${ }^{2}$ Georg Lukács, Histoire et conscience de classe: Essai de dialectique marxiste, trad. fr. K. Axelos et J. Bois, Paris, Éditions de Minuit, 1960, p. 117.

${ }^{3}$ Siegfried Kracauer, "La Photographie », dans Id., L'Ornement de la masse, op. cit., p. $36[\mathrm{FZ}, 28.10 .1927]$. 
nécessaire qu'on peut aussi les imaginer agencées autrement $»^{1}$. Cette contingence est aussi celle du présent des petits-enfants, d'autant plus qu'aujourd'hui la réalité, même contemporaine, n'est plus perçue qu'à travers les photographies des journaux illustrés; la réalité est devenue elle-même entièrement photogénique : «Car le monde lui-même s'est constitué un "visage photographique" $»^{2}$.

Mais en introduisant à la fin du texte le cinéma, Kracauer va dépasser le point de vue d'une simple critique de la modernité inspirée par la philosophie de la vie et la philosophie de l'existence. En effet, le stade ultime de la dissociation ouvre la voie à une recomposition générale d'un monde qui a atteint le point extrême de la désorganisation, et, selon Kracauer, ce pourrait être là le rôle du cinéma : préfigurer un ordre alternatif, un monde recomposé, casser la surface de la mauvaise réalité. Cette perspective de recomposition n'est évidemment pas présente chez Bergson et dans la philosophie de la vie. Kracauer greffe sur ce schéma bergsonien une perspective émancipatrice qui se situe sur un terrain collectif: le film pourrait être annonciateur d'une appropriation vivante de leur histoire par les hommes, qui redonnerait un sens à ce monde dissocié, en attente, un sens comparable à celui que représente, au plan individuel, le souvenir. La critique d'un capitalisme mortifère dominé par la ratio devient ainsi un processus dynamique.

\section{Un regard désenchanteur}

Le cinéma apparaît comme un instrument de connaissance critique, c'est-àdire une connaissance qui préfigure une alternative au monde réifié. La critique est plus qu'un simple lamento sur l'état désespérant du monde, mais s'inscrit dans une dynamique du désenchantement qu'il s'agit de mener à son terme, comme Kracauer l'explique dans l'essai intitulé L'Ornement de la masse $^{3}$.

Le cinéma est une source d'inspiration pour Kracauer, parce que non seulement il contribue à orienter la pensée vers les phénomènes, vers la réalité morcelée et chaotique du monde moderne, mais aussi parce qu'il met cette réalité en mouvement.

\footnotetext{
${ }^{1}$ Ibid., p. 44.

${ }^{2}$ Ibid., p. 46.

${ }^{3}$ Siegfried Kracauer, " L'Ornement de la masse », dans Id., L'Ornement de la masse, op. cit., p. 60-70.
} 
Les critiques des années 1920 et 1930 font apparaître très clairement cette double dimension à la fois mimétique mais aussi critique du cinéma. Tout en montrant la réalité désenchantée, le cinéma introduit un point de vue ironique : en nous révélant cette réalité de la ville moderne, il la met à distance, il expose son côté absurde. Cette ironie a une dimension métaphysique : elle expose la vanité d'une réalité qui se donne comme seul horizon possible. Kracauer se propose ainsi d'écrire une métaphysique du film qui analyserait cette dimension ironique, dont le ciné-œil de Vertov est à ses yeux un exemple paradigmatique. Tout comme l'ironie romantique, le regard de Vertov s'en prend à l'arrogance de ce qui se donne pour la réalité, et Vertov nous rappelle que toute cette belle organisation, qui est aussi l'organisation de la ville, est promise à la mort ${ }^{1}$. Il n'est pas sûr que l'intention de Vertov, pour qui le cinéma semble surtout un moyen pour la masse de prendre conscience d'elle-même et de s'approprier le monde mécanisé et dynamique de la métropole moderne, soit exactement celle-là, mais pour Kracauer, le cinéma apparaît aussi comme un contrepoids au monde mécanisé qu'il met pourtant en scène ${ }^{2}$. Kracauer évoque ainsi la manière dont René Clair explore d'une manière ludique la réalité du monde rationalisé, et opère une libération de la matière, paradoxalement rendue possible par la technique ${ }^{3}$.

Ce double mouvement de traversée de la réalité et de critique de celleci rappelle le principe de la " construction dans le matériau » développé dans Les Employés, qui est la grande contribution de Kracauer à l'ethnographie urbaine. Le film est souvent présenté par Kracauer à l'époque comme un moyen de connaissance de la réalité sociale (un exemple paradigmatique étant le film Lonesome/Solitude du cinéaste et anthropologue Paul Fejos, qui raconte une rencontre entre un ouvrier et une standardiste à Coney Island ${ }^{4}$ ). Le Kulturfilm urbain et contemporain pour lequel Kracauer ne cesse de

1 Siegfried Kracauer, «Der Mann mit dem Kinoapparat: Ein neuer russischer Film », dans Id., Kleine Schriften zum Film 1928-1931, op. cit., p. 249 [FZ, 19.5.1925].

${ }^{2}$ Siegfried Kracauer, « Artistisches und Amerikanisches », dans Id., Kleine Schriften zum Film 1921-1927, op. cit., p. 199 [FZ, 29.1.1926].

${ }^{3}$ Siegfried Kracauer, "Rationalisierung und Unterwelt : Ein Filmbericht », dans Id., Kleine Schriften zum Film 1932-1961, op. cit., p. 22 [FZ, 27.1.1932].

${ }^{4}$ Siegfried Kracauer, «Lonesome : Ein guter Film », dans Id., Kleine Schriften zum Film 1928-1931, op. cit., p. 236-238 [FZ, 9.4.1929]. 
plaider ${ }^{1}$, et qui ne mènerait plus le public dans des régions exotiques, mais se focaliserait sur des thèmes tels que les luttes sociales ou l'existence des employés ${ }^{2}$, est l'équivalent cinématographique des Employés. Kracauer est tout particulièrement intéressé par les documentaires sur la ville, comme le film d'Heinrich Hauser sur Chicago, ou comme Housing Problems de John Grierson.

En ce sens, et même s'il est un art de la surface, on peut aussi considérer que le film casse cette surface et - comme le dit explicitement Kracauer - qu'il va sous celle-ci ${ }^{3}$, dans le sens où il ne se contente pas de dupliquer la façon dont nous la percevons communément, ne serait-ce que parce qu'il est capable de montrer le monde sous des perspectives inhabituelles, de révéler «l'exotisme du quotidien », qui était aussi — de façon tout à fait explicite - le sujet de l'enquête des Employés. Le cinéma opère un effet « d'estrangement », sans lequel il ne saurait y avoir de connaissance.

Il est essentiel pour Kracauer que le cinéma opère à partir de la réalité et dans la réalité. Le cinéma peut afficher une «tendance », développer un discours critique, conformément à sa capacité d'estrangement, mais la critique doit passer, comme par exemple chez Capra ${ }^{4}$, par les objets de la surface, par les petits éléments du monde matériel, et non pas par une construction discursive inscrite dans le scénario. Le cinéma ne critique pas la réalité de façon argumentative, mais en tant qu'il la montre, qu'il la fait parler : c'est pourquoi le film s'accommode mal de ce didactisme dont fait preuve, selon Kracauer, Balász dans son film Zehnmarkschein ${ }^{5}$. Dans les années 1930, Kracauer approuve l'évolution de Jean Vigo dans l'Atalante. En atténuant sa critique sociale explicite, Vigo fait passer au premier plan les détails, les petites choses matérielles, les éléments épisodiques ${ }^{6}$. C'est ici

${ }^{1}$ Voir par exemple Siegfried Kracauer, « Über den Umgang mit Tieren. Anläßlich eines Jagdfilmes », dans Id., Kleine Schriften zum Film 1928-1931, op. cit., p. 399401 [FZ, 28.9.1930].

${ }^{2}$ Voir par exemple Siegfried Kracauer, «Internationaler Tonfilm », dans Id., Kleine Schriften zum Film 1928-1931, op. cit., p. 475-479, p. 478 [Europäische Revue, Vol. 7, Mars 1931, p. 228-231].

3 Voir par exemple Siegfried Kracauer «Der grosse Gabbo », dans Id., Kleine Schriften zum Film 1928-1931, op. cit., p. 358-360 [FZ, 22.5.1930].

${ }^{4}$ Siegfried Kracauer, «Frank Capra», dans Id., Kleine Schriften zum Film 19321961, op. cit., p. 271-273 [NZZ, 11.6.1939].

${ }^{5}$ Siegfried Kracauer, "Abenteuer eines Zehnmarkscheines », dans Id., Kleine Schriften zum Film 1921-1927, op. cit., p. 275-276 [FZ, 5.12.1926].

${ }^{6}$ Siegfried Kracauer, «Wiedersehen mit alten Filmen », dans Id., Kleine Schriften zum Film 1932-1961, op. cit., p. 299-333 [BNZ, 1.2.1940]. 
qu'intervient la question complexe du montage, et Kracauer essaie d'éviter deux écueils : un montage excessif qui plaque un discours argumentatif sur la réalité et n'opère plus dans cette réalité, et un montage insuffisant, qui ne fait pas parler la réalité, mais se contente d'en reproduire la surface usuelle.

Dans un premier temps, le cinéma soviétique semble incarner pour Kracauer ce réalisme critique qui traverse les objets, les fait parler, en respectant leur épaisseur matérielle. Ce qui manque au cinéma allemand (en particulier à Ruttmann, l'auteur de Berlin. Sinfonie einer Grosstadt) par rapport au cinéma russe, c'est précisément cette capacité à faire parler les objets de la surface urbaine: le film de Ruttmann propose un montage purement ornemental et incarne l'esprit de la nouvelle objectivité. Par la suite, il prend ses distances par rapport au cinéma soviétique, dès la fin de la République de Weimar et encore plus dans ses œuvres ultérieures, où il reproche à ce cinéma son goût immodéré pour le symbole, qui s'éloigne des choses au profit de significations qui leur sont imposées de l'extérieur.

\section{Une expérience utopique}

Le cinéma, médium au potentiel critique — même si évidemment ce potentiel n'est pas toujours réalisé —, apparaît même parfois comme le lieu d'une sorte d'expérience utopique anticipant le terme de ce processus de désenchantement, dont il est l'expression, et au bout duquel il y a, selon L'Ornement de la masse, « la vie humaine », l' ' humain tel qu'il est en vertu de la raison $»{ }^{1}$. Car si le cinéma fait contrepoids au monde mécanisé, c'est aussi parce qu'il met la surface en mouvement, et préfigure donc cet ordre raisonnable substantiel évoqué à la fin de L'Ornement de la masse, cette Heimat universelle, dont la vision s'est déposée dans les contes de fée, et qui est le pays du vagabond Charlot, à qui Kracauer consacre de nombreux textes. Une autre figure importante est celle de Greta Garbo, qui incarne aux yeux de Kracauer une nature humanisée ${ }^{2}$. La dimension utopique du cinéma est évoquée à la fin du texte sur la photographie, comme on l'a vu, avec cette perspective d'une recomposition. En tant qu'art de la recomposition, le cinéma retrouve alors une propriété que Kracauer associe à la modernité : la capacité d'improvisation. Cette notion exprime chez Kracauer toute l'ambi-

\footnotetext{
${ }^{1}$ Siegfried Kracauer, « L'Ornement de la masse », dans Id., L'Ornement de la masse, op. cit., p. 71.

${ }^{2}$ Siegfried Kracauer, "Greta Garbo eine Studie », dans Id., Kleine Schriften zum Film 1932-1961, op. cit., p. 50-154 [FZ, 25.2.1933].
} 
valence de la modernité. En effet, d'un côté l'improvisation est liée au désordre: dans un univers ordonné, il n'y a pas d'improvisation. Celle-ci n'est possible qu'à l'âge du déracinement. D'un autre côté, cette faculté d'improvisation renvoie à une mobilité moderne productive. Les clowns acrobates, dont les figures libres - donnant l'apparence de l'improvisation - semblent vaincre la pesanteur, abolir toute contrainte physique, formulent une promesse d'émancipation de la nature ${ }^{1}$. L'improvisation est mouvement créateur, et le cinéma introduit précisément par rapport à la photographie une dimension de mobilité :

Le cinéma nous a habitué à ne plus considérer les objets depuis un point fixe, mais, en glissant pour les contourner, à avoir le libre choix de nos perspectives. Ce que peut le cinéma, fixer des objets en mouvement, est interdit à la photographie ${ }^{2}$.

Dans le texte «Monde du calicot», qui relate une visite dans l'univers de carton-pâte des studios de Babelsberg, Kracauer se montre fasciné par l'aptitude du cinéma à fragmenter et recomposer le monde, et donc, quelque part, à le remettre en mouvement, même si c'est sous la forme de simulacres ${ }^{3}$. Le texte «Culte de la distraction » (« Kult der Zerstreuung ») consacré à la culture de masse, et plus particulièrement aux temples du divertissement que sont les grandes salles de cinéma va absolument dans le même sens. Kracauer y explique que la distraction (Zerstreuung) n'a de sens que dans l'improvisation ${ }^{4}$. La culture moderne, tournée vers l'extériorité, et l'étourdissement qu'elle suscite, a la sincérité pour soi, et les masses à qui s'adresse cette culture de l'improvisation, ne sont pas loin de la vérité. Ce n'est pas elle qui menace la vérité. Celle-ci n'est menacée que par l'affirmation naïve de valeurs culturelles devenues irréelles.

De ce point de vue aussi, il y a une sorte d'homologie entre le cinéma et la ville, car la ville est aussi riche en lieux d'improvisation, de recomposition comme les gares, les passages. Le visiteur des «bars dans le sud»,

${ }^{1}$ Siegfried Kracauer, « Acrobate-Bö », dans Id., Rues de Berlin et d'ailleurs, op. cit., p. 177-182.

${ }^{2}$ Siegfried Kracauer, "Photographiertes Berlin», dans Id., Essays, Feuilletons, Rezensionen 1932-1965 (Werke 5.3, herausgegeben von Inka Mülder-Bach; unter Mitarbeit von Sabine Biebl), Berlin, Suhrkamp, 2011, p. 310 [FZ,15.12.1932].

${ }^{3}$ Siegfried Kracauer, « Monde du calicot», dans Id., Le Voyage et la danse, op. cit., p. 33-40 [FZ, 28.1.1926].

${ }^{4}$ Siegfried Kracauer, "Culte de la distraction », dans Id., L'Ornement de la masse, op. cit., p. 286-291. 
que Kracauer compare à des « petits ports minuscules, d'où on peut prendre le départ », en sort avec une autre vision de la réalité, car

les éléments d'une existence stable y sont amarrés sans égard à leur rang, les structures des palais ne résistent pas aux reflets déformants des miroirs. Ainsi celui qui les quitte perd le sens des proportions de la vie qu'il laisse derrière lui. Elle se brise pour lui en une multitude de morceaux isolés, à partir desquels il peut improviser les fragments d'une autre vie. La valeur des villes se mesure au nombre des lieux qu'elles réservent à l'improvisation ${ }^{1}$.

On pourrait ici également évoquer ici le chapitre final du roman Ginster, qui se déroule à Marseille. Kracauer recourt, dans la première théorie du film, esquissée à Marseille en 1940/41 et à propos du film La Rue, à une image nietzschéenne qui explicite cette dialectique du désordre et de la recomposition : " Ce qui est chaotique pourra peut-être donner naissance à des étoiles $\gg{ }^{2}$. Lieu d'aliénation et de solitude, les villes sont donc également pour Kracauer le site d'une dynamique positive. Le déracinement n'est donc pas seulement un déchirement: Genêt souhaite vivre dans l'incognito, recherche cette impersonnalité des halls d'hôtel. Le renouvellement permanent de la population fait justement le charme de l'immeuble de rapport, que Kracauer, qui semble habiter dans l'un d'entre eux, préfère au pavillon individuel : "Car ce qu'il y a de merveilleux dans cet immeuble de rapport, c'est justement que, comme un quartier portuaire, il accueille chaque jour de nouveaux arrivants, et qu'il ne se contente pas de donner sur la rue, mais est lui-même une rue $»^{3}$.

\section{De la recomposition au sauvetage}

Comme on l'a dit, il était essentiel pour Kracauer, même dans le cadre d'un discours critique, de traverser les choses matérielles. On observe toutefois au début des années 1930 une inflexion de la perspective. En juillet 1932, Kracauer se met à réfléchir sur ce qu'il caractérise comme l'intention initiale de la photographie, qui caractériserait ce médium en tant que tel, et le

${ }^{1}$ Siegfried Kracauer, « Bars dans le sud », dans Id., Rues de Berlin et d'ailleurs, op. cit., p. 89.

${ }^{2}$ Siegfried Kracauer, «Marseiller Entwurf», dans Id., Theorie des Films. Die Errettung der äußeren Wirklichkeit, op. cit., p. 699.

3, Siegfried Kracauer, "Mietshaus im Berliner Westen», dans Id., Essays, Feuilletons, Rezensionen 1928-1931, op. cit., p. 464 [FZ, 6.3.1931]. 
distinguerait des autres. L'exposition qui est l'occasion de cette réflexion (Berliner Film- und Photo-Schau") présentait en particulier la «vue de la fenêtre" de Niepce (considérée comme le tout premier cliché photographique de l'histoire). Ce cliché de Niepce inspire à Kracauer l'idée que la photographie peut aussi sauver les choses promises à la mort, les délivrer de la malédiction qui les frappe, sauver le présent de l'oubli : les photographies les plus anciennes n'avaient pas d'autre ambition que de sauver le fugitif, car il s'agissait de capter le transitoire, et de le montrer en tant que tel, sans le réifier, le transfigurer, le figer. La photographie ne fait donc pas qu'enregistrer une négativité, reproduire un ordre mortifère, qui était la condition d'une recomposition dynamique. Les choses du monde désenchanté valent en quelque sorte pour elles-mêmes, tout en étant contingentes, elles sont légitimes, porteuses d'une positivité. La seconde partie du texte est consacrée au cinéma. Kracauer suggère que, comme dans le cas de la photographie, le potentiel initial spécifique à ce médium a été galvaudé : la volonté d'ennoblir le cinéma, de lui conférer une qualité artistique, ainsi que les progrès de la technologie ont conduit à une perte de substance, à l'étouffement du potentiel de ce médium ${ }^{2}$, cette dénonciation de l'embourgeoisement du cinéma étant une constante dans l'œuvre de Kracauer.

Il y a alors de plus en plus dans les textes de Kracauer un lien très affirmé entre le cinéma et la vie en tant que telle, et la promesse de recomposition passe au second plan - ce qui explique peut-être la critique du montage. Le cinéma apparaît comme une victoire sur la mort, et cet élément précaire et fugitif qui se voit sauvé est la vie dans ce qu'elle a d'impalpable. Dans la première «théorie du film», rédigée à Marseille en 1940/41 dans des circonstances dramatiques, Kracauer parle pour désigner cette vie précaire de «réalité», celle-ci étant définie comme la «strate fondamentale » (« Grundschicht »), c'est-à-dire la réalité physique, indépendante de toute intentionnalité ou finalité humaine, et qui n'était jusque-là pas visible, parce qu'elle était voilée par des visions théologiques du monde, et parce qu'il n'existait pas de technique d'enregistrement de cette strate, c'està-dire pas de photographie. On retrouve l'idée de décentrement et de mobilité, car le cinéma est l'expression d'une époque qui n'est plus inscrite

\footnotetext{
${ }^{1}$ Siegfried Kracauer, «An der Grenze des Gestern : Zur Berliner Film- und photoSchau ", dans Id., Kleine Schriften zum Film 1932-1961, op. cit., p. 76-82 [FZ, 12.7.1932].

${ }^{2}$ Ibid., p. 81.
} 
dans la «structure statique » d'une tradition, prise dans un ordre englobant ${ }^{1}$. La « réalité » est le lieu de l'aléatoire, de l'infini.

Ces éléments matériels que le cinéma arrache à la disparation, ce sont par exemple les configurations précaires de la ville, qui apparaissent comme emblématiques de la réalité qui se donne à voir grâce au cinéma. Ainsi, le film sonore a l'intérêt de pouvoir capter le brouhaha de la rue ${ }^{2}$, et ce qu'on pourrait appeler la surface sonore et anonyme de la ville, une réalité qui n'est pas intentionnelle. Kracauer évoque à nouveau dans ce manuscrit la rue et ses constellations mouvantes, mais aussi les gares, les ports, les fêtes foraines, etc. C'est grâce au dispositif mécanique et technique de la photographie que l'homme accède au sens où Kracauer l'entend. La part d'impersonnalité et de neutralité que comporte toujours une représentation photographique est ce qui permet d'accéder à cette "strate fondamentale » qui constitue selon Kracauer le terrain d'élection du cinéma.

À travers la valorisation de l'expérience de la réalité dans le cinéma, il ne s'agit donc pas de retrouver ce qui serait une expérience " authentique » du monde, à la façon de la «philosophie de la vie » et de sa critique d'un monde mécanisé. C'est bien l'âge de la science, dont les médias photographiques sont le produit, qui fait émerger la vie, la rend visible. Kracauer prend sur ce point Simmel à contre-pied, et ce de façon tout à fait explicite. Selon Simmel, en effet, Rembrandt allait dans ses portraits au-delà de la surface optique des choses, il saisissait le sens du phénomène, sa " nécessité intérieure » alors que la photographie n'est qu' « une reproduction littérale » ${ }^{3}$. Kracauer note aussi cette tendance de la photographie à la littéralité, mais à l'inverse de Bergson et de Simmel, Kracauer considère pour sa part cette tendance de la photographie à l'impersonnel comme porteuse d'un potentiel positif, alors que chez Simmel elle sert exclusivement à valoriser l'art du portrait pictural. Pour Simmel, le vital et l'impersonnel sont en opposition, alors qu'il se confirme que pour Kracauer c'est la neutralité impersonnelle de la technique qui nous donne accès à une réalité précaire et mobile de la vie, qui se déploie dans un monde spatialisé et désenchanté.

${ }^{1}$ « Par exemple, la prédilection que manifestent aujourd'hui tant de gens pour les amples perspectives et les vues panoramiques pourrait bien remonter à une époque moins dynamique que la nôtre », Siegfried Kracauer, Théorie du film: La rédemption de la réalité matérielle, trad. fr. D. Blanchard et C. Orsoni, Paris, Éditions Flammarion, 2010, p. 36-37.

${ }^{2}$ Siegfried Kracauer, "Tonbildfilm », dans Id., Kleine Schriften zum Film 19281931, op. cit., p. 122-125 [FZ, 12.10.1928].

${ }^{3}$ Georg Simmel, «Le Problème du portrait », dans Id., Philosophie de la modernité, op. cit., p. 150-151. 
S'il s'agit plutôt de sauver ces configurations précaires que d'aller vers une recomposition, ce geste de sauvetage a une dimension subversive, y compris au plan politique (au moins dans la première version de la Théorie $d u$ film). Lorsque dans Charlot machiniste, le vagabond sème le trouble dans un théâtre, il semble dire que " cela ne va pas sans nous, la racaille $»^{1}$. Le cinéma, divertissement qui expose le corps et s'adresse à lui, est aussi le médium d'un renversement social. Contemporain de l'avènement des masses, il présente une affinité certaine avec le "matérialisme». C'est pourquoi le « film d'art», vendu au théâtre, et qui neutralise le réalisme du cinéma, se voit comparé par Kracauer au "prolétaire renégat», qui a adopté les convictions de la classe dominante.

Il semble que la scène de la fête des morts dans Que viva Mexico d'Eisenstein était pour Kracauer emblématique du pouvoir de vie et de rébellion du cinéma :

Les Indiens s'y moquent des généraux, des prêtres et de la Mort, s'enivrent, dansent sur les tombes... et la vie continue. Cette grandiose épopée du peuple mexicain devait se terminer sur l'image d'un enfant qui arrache de sa figure le masque traditionnel de la mort et rayonne de la joie de vivre ${ }^{2}$.

\section{Le flux de la vie}

On retrouve dans ses grandes lignes la structure qui vient d'être décrite dans la seconde «théorie du film »: pour la première fois dans l'histoire de l'art, le cinéma nous met selon Kracauer en contact avec la substance matérielle de la réalité, le «flux de la vie». Ce flux est le «continuum de l'existence physique », la «nature à l'état brut » : c'est par exemple le tremblement des feuilles agitées par le vent, la surface d'une flaque trouble. Cette notion de vie ou de monde matériel ne peut en effet advenir que quand le monde est désenchanté, n'est plus perçu à travers le filtre de systèmes de croyance : «La vie en tant qu'entité puissante [...] est un concept relativement récent $»^{3}$.

L'objet du cinéma, c'est donc à nouveau la vie aléatoire, éphémère, vibrante, celle des rues de la ville, en tant que site éminent de la contingence.

${ }^{1}$ Siegfried Kracauer, «Marseiller Entwurf», dans Id., Theorie des Films. Die Errettung der äußeren Wirklichkeit, op. cit., p. 557.

${ }^{2}$ Tiré de l'article « Histoire du film inachevé d'Eisenstein », dans Revue du cinéma, octobre 1948 (fiche tirée des brouillons de la Théorie du film).

${ }^{3}$ Siegfried Kracauer, Théorie du film, op. cit., p. 251. 
À propos des premiers films des frères Lumière, Kracauer note : «On avait là la vie dans ses moments les moins maîtrisables et les plus innocents, un fouillis de formes fugitives et à jamais enfuies, que seule la caméra pouvait saisir $\rangle^{1}$. Le film a donc une affinité avec la réalité non organisée, l'aléatoire, l'infini, l'indéterminé. Kracauer voit de ce point de vue une analogie avec le roman moderne (Virginia Woolf, Marcel Proust) tel que le présente Erich Auerbach, même si le roman moderne n'explore pas le continuum matériel, mais le continuum spirituel de la réalité ${ }^{2}$.

Si cette vie devient visible, c'est parce que le monde est désenchanté, n'est plus informé par des catégories théologiques ou post-théologiques. La sélection de photographies opérée par Kracauer pour illustrer son ouvrage est à cet égard significative: elle comporte plusieurs vues urbaines, qui reprennent certaines figures obsessionnelles des textes de ville, les ruines, le vide, la mélancolie : une photographie d'Atget qui montre une rue déserte, ainsi qu'une vue - tirée de Housing problems — sur des taudis misérables, un cliché de Cartier-Bresson montrant des enfants dans les ruines. Kracauer évoque lui-même explicitement le climat mélancolique des photographies de Marville ou Atget $^{3}$. Ces figures urbaines renvoient clairement au sentiment d'un vide normatif, qu'il s'agit d'affronter, et dont la face positive est précisément la possibilité d'une expérience de la réalité. Le cinéma néoréaliste italien, qui correspond assez bien à l'idée du cinéma que Kracauer défend dans la Théorie $d u$ film, avait une prédilection certaine pour les paysages urbains mélancoliques, les " espaces quelconques » qui, selon Gilles Deleuze, «s'opposent aux espaces déterminés de l'ancien réalisme » ${ }^{4}$.

Si le cinéma permet l'exploration du flux de la vie, c'est qu'il capte le mouvement d'une façon en partie impersonnelle, comme l'avait suggéré Proust $^{5}$. Pour démontrer l'existence de cette composante impersonnelle dans le regard photographique, Kracauer s'appuie sur un passage de Du côté de Guermantes où le narrateur, apercevant sa grand-mère en entrant dans une pièce, la voit le temps d'un instant comme la vieille femme qu'elle est, dans une perception dépouillée de toute aura affective et que Proust compare à une

\footnotetext{
${ }^{1}$ Ibid., p. 66.

${ }^{2}$ Erich Auerbach, Mimesis, trad. fr. C. Heim, Paris, Éditions Gallimard, 1977.

${ }^{3}$ Siegfried Kracauer, Théorie du film, op. cit., p. 46-47.

${ }^{4}$ Gilles Deleuze, L'Image-mouvement, Paris, Éditions de Minuit, 1981, p. 286.

${ }^{5}$ Pour prévenir certains malentendus sur le réalisme de Kracauer, il faut noter que l'image photographique ou filmique n'est jamais neutre, mais qu'elle résulte d'un équilibre entre la tendance réaliste ou réceptrice et une tendance « formative».
} 
photographie ${ }^{1}$. On reconnaît dans cette opposition entre une image affective et l'image objective, impitoyable, l'argumentation du texte de 1927 sur « La Photographie », qui opposait souvenir vivant et image photographique. Cependant, par rapport à 1927, la perspective s'est infléchie : la vision d'une appropriation collective de l'histoire, qui permettrait, comme le fait le souvenir à l'échelle individuelle, de recomposer les fragments de la réalité que la photographie inventorie, semble ici absente. Il ne s'agit plus d'en appeler à un dépassement du stade que représente l'image photographique en tant qu'image-espace. Au contraire, c'est la photographie qui permet de capter la fragilité du vivant, sa précarité, et l'ombre de la mort qui pèse sur lui (et face à laquelle il n'y a plus de consolation religieuse). On voit donc déjà ici comment le cinéma pourra devenir dans History (que Kracauer présentera comme un prolongement de la Théorie du film) une sorte de modèle cognitif. Comme le regard de l'historien dans History, historien qui est souvent un étranger, un exilé, le cinéma produit naturellement cet effet d' « estrangement ».

En tant que moyen de connaissance, le cinéma s'inscrit donc bien dans une dynamique critique de la modernité, dans la mesure où celle-ci détruit les mythes et les certitudes établies. Toutefois, il est vrai que Kracauer concède à la philosophie de la vie et à sa critique de la modernité mécanisée que l'esprit moderne est un esprit d'abstraction, qui peut nous éloigner du contact matériel avec les choses sensibles: le visage de la science est - dans la Théorie du film - double : elle contribue à la destruction des mythes, mais au prix d'une abstraction. Avec le cinéma, la modernité sécrète en quelque sorte son contrepoison : le film est « une roue papillotante de la vie $»^{2}$, et est en mesure de répondre à la « soif de vie $»^{3}$ suscitée par le développement de l'abstraction : le cinéma « donne la capacité de rétablir un contact "sensuel et immédiat" avec "la vie" »". La leçon de la Théorie $d u$ film est donc que la modernité a la capacité de lutter contre sa propre abstraction, d'inventer une image vivante d'elle-même.

Il est donc essentiel de comprendre que ce constat du désenchantement n'implique pas plus qu'avant la nostalgie d'un ordre antérieur, même s'il s'inscrit sans doute aussi en faux contre un certain modernisme affirmatif. Il

${ }^{1}$ Marcel Proust, Le Côté de Guermantes, I, dans Id., À la recherche du temps perdu, II, Paris, Éditions Gallimard, 1988, p. 438-439.

${ }^{2}$ Siegfried Kracauer, Théorie du film, op. cit., p. 252.

${ }^{3}$ Ibid., p. 249.

4 Ibid., p. 252. Kracauer cite ici l'ouvrage de Wolfgang Wilhelm intitulé Die Auftriebwirkung des Films (Bremen, Geist, 1940). 
ne s'agit ainsi pas de nier la part d'ombre du développement scientifique, non seulement l'abstraction, mais aussi la possibilité d'industrialiser la mort (dans les abattoirs et les camps d'extermination). Il n'échappe pas à Kracauer que la modernité offre l'accès à la vie et développe en même temps des moyens inédits pour la détruire et la réflexion sur l'horreur occupe une place non négligeable dans la Théorie $d u$ film $^{1}$, une idée centrale étant que le cinéma nous donne aussi les moyens d'affronter cette horreur symbolisée par la face de Méduse. Sans doute faut-il voir là une réplique à Adorno et son interdit de l'image, lié à l'idée selon laquelle Auschwitz serait irreprésentable ou indicible. À cela, Kracauer oppose une comparaison entre le cinéma et le bouclier de Persée, qui reflète l'image de la méduse, et permet de maîtriser la terreur qu'elle nous inspire. À Adorno s'oppose aussi le refus de voir la modernité uniquement sous l'angle de la catastrophe, de l'aliénation, de promesses non tenues, qui se sont retournées en oppression du sujet, et que seule une dialectique négative permettrait de réactiver.

Mais si on peut avoir l'impression que le «flux de la vie» ne s'achemine vers aucune totalité ou réconciliation, Kracauer parle de façon assez provocante à propos du cinéma de « rédemption » des choses matérielles : il y a donc un horizon de sens qui ne s'épuise pas dans le «flux de la vie», et qu'il s'agit d'atteindre à travers le monde matériel. C'est ce qu'essaie de préciser l'ouvrage posthume History, qui étend la notion de "réalité »cette réalité captée par le cinéma, ou caméra-réalité - au champ historique, et l'associe à nouveau à la vie en introduisant la notion de «Lebenswelt» :

La « caméra-réalité » — cette sorte de réalité vers laquelle le photographe, où le caméraman, dirige ses objectifs - présente plutôt tous les caractères du «Lebenswelt ». Elle comporte des objets inanimés, des visages, des foules, des gens qui s'entrecroisent, qui souffrent et qui espèrent; son sujet de prédilection, c'est la vie dans sa plénitude, la vie telle que nous la vivons communément ${ }^{2}$.

Comme le notent Philippe Despoix et Nia Perivolaropoulou, les composantes principales de ce flux de la vie et de ce monde de la vie sont « le fugitif, l'indéterminé, l'amorphe, le singulier, ce qui est sans nom ${ }^{3}$.

${ }^{1} C f$. Olivier Agard, « Cinéma et horreur chez Siegfried Kracauer », dans Germanica, $\mathrm{n}^{\circ} 37,2005$, p. $107-120$.

${ }^{2}$ Siegfried Kracauer, L'Histoire: Des avant-dernières choses, trad. fr. C. Orsoni, Paris, Éditions Stock, 2006, p. 117.

3 Philippe Despoix \& Nia Perivolaropoulou, «Postface: «"L'Histoire est un amusement de vieillard" ", dans Siegfried Kracauer, L'Histoire, op. cit., p. 302. 
Pour Kracauer, il s'agit, dans cet espace intermédiaire, cette «antichambre » que constitue l'univers historique, d'éviter ce double écueil du « transcendantalisme» et de 1 '« immanentisme », c'est-à-dire de «reconnaître l'importance éventuelle de vérités philosophiques avec leur prétention à une validité objective », tout en étant conscient de «leurs limites en termes d'absolu et de pouvoir de contrôle »' ${ }^{1}$. Il n'y a plus un sens absolu qui s'impose à nous, mais un sens éclaté et multiple, fragmentaire et diffus, à reconstruire par les individus, des signes polysémiques et opaques, des symboles ouverts, comme les trois arbres dans la recherche du temps perdu, à propos desquels Proust écrit :

Je vis les arbres s'éloigner en agitant leurs bras désespérés, semblant me dire : ce que tu n'apprends pas de nous aujourd'hui tu ne le sauras jamais. Si tu nous laisses retomber au fond de ce chemin d'où nous cherchions à nous hisser jusqu'à toi, toute une partie de toi-même que nous t'apportions tombera pour jamais au néant ${ }^{2}$.

Le cinéaste est celui qui arrive à faire parler les objets du monde, à les replacer dans un horizon universel, d'où ils font sens pour nous.

${ }^{1}$ Siegfried Kracauer, L'Histoire, op. cit., p. 291.

${ }^{2}$ Marcel Proust, $A$ l'ombre des jeunes filles en fleur, dans $I d$., À la recherche $d u$ temps perdu, I, Paris, Éditions Gallimard, 1987, p. 717. 